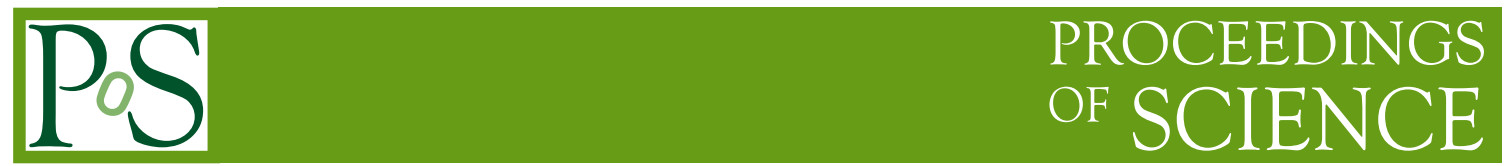

$B_{s} \rightarrow \mu^{+} \mu^{-}$in the SM and $\bar{B} \rightarrow X_{s} \gamma$ in the 2HDM at NNLO in QCD

\title{
Thomas Hermann*
}

Institut für Theoretische Teilchenphysik

Karlsruhe Institute of Technology (KIT)

D-76128 Karlsruhe, Germany KIT

E-mail: t.hermannekit.edu

In this contribution the QCD next-to-next-to-leading order (NNLO) matching coefficients to the branching ratio $B_{S} \rightarrow \mu^{+} \mu^{-}$in the Standard Model (SM) and to $\bar{B} \rightarrow X_{s} \gamma$ in the Two Higgs Doublet Model (2HDM) are discussed. In both cases a three-loop matching between the full and effective theory was performed, which leads to a significant reduction of the scale uncertainty.

11th International Symposium on Radiative Corrections (Applications of Quantum Field Theory to Phenomenology)

22-27 September 2013

Lumley Castle Hotel, Durham, UK

*Speaker. 


\section{Introduction}

Together with direct searches at the LHC, rare B meson decays are very important for the search of physics beyond the Standard Model (SM). Therefore, it is necessary to provide precise theory predictions to those decays.

The decay $B_{s} \rightarrow \mu^{+} \mu^{-}$yields important constraints on extensions of the SM. Recently, $\mathrm{LHCb}$ and CMS have provided first measurements of the branching ratio [1-3] and their combined result reads [4]

$$
\overline{\mathscr{B}}\left(B_{s} \rightarrow \mu^{+} \mu^{-}\right)=(2.9 \pm 0.7) \times 10^{-9} .
$$

Previous upper limits can be found in Refs. [5-9]. In the future a significant reduction of the experimental uncertainties is expected. On the theory side the leptonic decay constant $f_{B_{s}}$ was the dominant uncertainty in the last years. Recent progress in the determination of $f_{B_{s}}$ from lattice calculations [10-15] provides a motivation for improving the perturbative ingredients, in particular the two-loop electroweak [16] and the three-loop QCD corrections [17]. In Section 2 some aspects of the three-loop matching are discussed, the full discussion can be found in [17].

The inclusive decay $\bar{B} \rightarrow X_{s} \gamma$ also provides very strong constraints on physics beyond the SM. Especially in the Two Higgs Doublet Model (2HDM) of type-II, where $\bar{B} \rightarrow X_{s} \gamma$ gives one of the highest exclusion limits for the charged Higgs boson mass $M_{H^{+}}$. Therefore it is worth to calculate the three-loop QCD corrections to the corresponding Wilson coefficients in the 2HDM, which was done in Ref. [18] and is shortly described in Section 3. Together with all the other NNLO QCD ingredients $[19,20]$ obtained for the SM prediction of $\mathscr{B}\left(\bar{B} \rightarrow X_{s} \gamma\right)$, it is now possible to give also for the $2 \mathrm{HDM}$ a consistent prediction at NNLO in QCD.

\section{2. $B_{S} \rightarrow \mu^{+} \mu^{-}$in the SM at NNLO in QCD}

A convenient framework for calculating the branching ratio $B_{s} \rightarrow \mu^{+} \mu^{-}$, is an effective theory derived from the SM by integrating out all heavy particles like the top quark, the Higgs boson and the massive electroweak bosons. The relevant effective Lagrangian for $B_{s} \rightarrow \mu^{+} \mu^{-}$reads

$$
\mathscr{L}_{\text {eff }}=\mathscr{L}_{\mathrm{QCD} \times \mathrm{QED}}(\text { leptons and five light quarks })+N \sum_{n} C_{n} Q_{n}+\text { h.c. },
$$

with $N=V_{t b}^{*} V_{t s} G_{F}^{2} M_{W}^{2} / \pi^{2}$. The necessary operators are

$$
\begin{aligned}
Q_{A} & =\left(\bar{b} \gamma_{\alpha} \gamma_{5} s\right)\left(\bar{\mu} \gamma^{\alpha} \gamma_{5} \mu\right), \\
Q_{S} & =\left(\bar{b} \gamma_{5} s\right)(\bar{\mu} \mu), \\
Q_{P} & =\left(\bar{b} \gamma_{5} s\right)\left(\bar{\mu} \gamma_{5} \mu\right) .
\end{aligned}
$$

In the SM only the Wilson coefficient $C_{A}$ matters, because contributions from $C_{S}$ and $C_{P}$ to the branching ratio are suppresed by $M_{B_{s}}^{2} / M_{W}^{2}$. In this case, a formula for the measured average timeintegrated branching ratio [21] reads

$$
\overline{\mathscr{B}}\left(B_{s} \rightarrow \mu^{+} \mu^{-}\right)=\frac{|N|^{2} M_{B_{s}}^{3} f_{B_{s}}^{2}}{8 \pi \Gamma_{H}^{s}} \beta r^{2}\left|C_{A}\left(\mu_{b}\right)\right|^{2}+\mathscr{O}\left(\alpha_{e m}\right)
$$


(a)

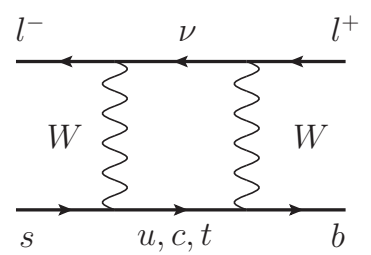

(b)

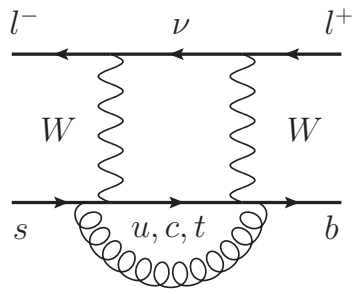

$(c)$

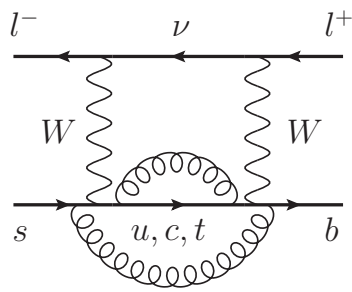

Figure 1: Sample $W$-boson box diagrams contributing to $C_{A}$.

with $r=\frac{2 m_{\mu}}{M_{B_{s}}}$ and $\beta=\sqrt{1-r^{2}} . \Gamma_{H}^{s}$ is the total width of the heavier mass eigenstate.

In the SM there are two types of diagrams contributing to $C_{A}=C_{A}^{W}+C_{A}^{Z}$, the W-boson box and the Z-boson penguin diagrams (see Fig. 1 and Fig. 3), which are discussed in the following. The one-loop contribution to $C_{A}$ has been calculated for the first time in Ref. [22] and two-loop QCD correction can be found in Refs. [23-26].

Sample diagrams to $C_{A}^{W}$ at one-, two and three-loop order are shown in Fig. 1. Since the up and charm quark masses are set to zero, $C_{A}^{W}$ can be written as

$$
C_{A}^{W}=C_{A}^{W, t}-C_{A}^{W, c},
$$

due to the unitarity of the CKM matrix.

The matching can be performed in two different ways, in $d=4-2 \varepsilon$ and in $d=4$ dimensions. In the first approach we set all light quark masses to zero, which leads to spurious infrared divergences in $\varepsilon$ in the full and effective theories which cancel out in the matching procedure. Due to the presence of those additional poles at intermediate steps, it is necessary to introduce an evanescent operator

$$
Q_{A}^{E}=\left(\bar{b} \gamma_{\alpha_{1}} \gamma_{\alpha_{2}} \gamma_{\alpha_{3}} \gamma_{5} s\right)\left(\bar{\mu} \gamma^{\alpha_{3}} \gamma^{\alpha_{2}} \gamma^{\alpha_{1}} \gamma_{5} \mu\right)-4 Q_{A},
$$

which vanishes in $d=4$ dimensions. With the mixing of $Q_{A}^{E}$ into $Q_{A}$, the Wilson coefficient of the evanescent operator gives a contribution to the Wilson coefficient $C_{A}$.

In the second approach we introduce small masses for the strange and bottom quark as regulators for the infrared divergences. The matching of the full and effective theory can be performed in $d=4$ dimensions, so without contributions from evanescent operators. After the matching it is possible to take the limits $m_{s} \rightarrow 0$ and $m_{b} \rightarrow 0$. We have used both methods and have obtained identical results for $C_{A}$.

In the SM three-loop vacuum integrals with two different mass scales have to be computed. Some classes of Feynman diagrams of this kind are known (e.g., Ref. [27]), nevertheless we follow the same strategy as in Ref. [28]. We expand the integrals in the limit $M_{W} \ll m_{t}$ and $M_{W} \approx m_{t}$. A combination of those expansions gives a very good approximation to the exact three-loop result and is sufficient for all practical purposes. For the calculation we used the programs QGRAF [29] to generate the Feynman diagrams, q2e and exp [30] for the asymptotic expansions [31] and MATAD [32], written in Form [33], for evaluation of the three-loop diagrams. 

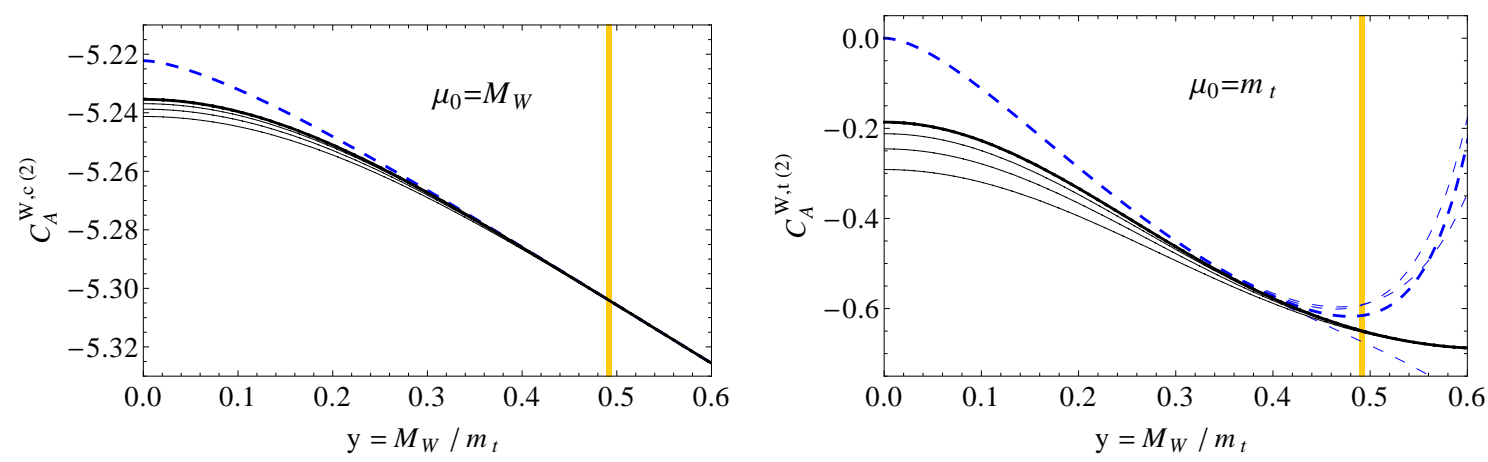

Figure 2: $C_{A}^{W,(2)}$ as a function of $y=M_{W} / m_{t}$ for the charm (left) and top quark sector (right). The (blue) dashed lines are obtained in the limit $y \ll 1$, and the (grey) solid line for $y \approx 1$. Thinner lines contain less terms in the expansions. The physical region for $y$ is indicated by the (yellow) vertical band.

(a)

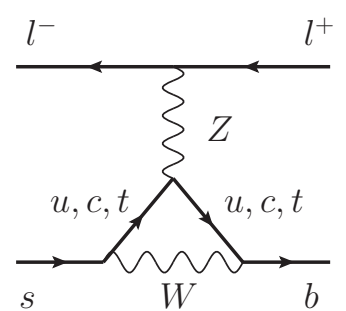

(b)

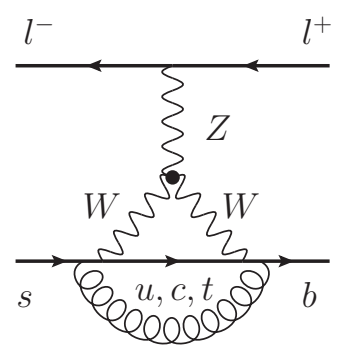

(c)

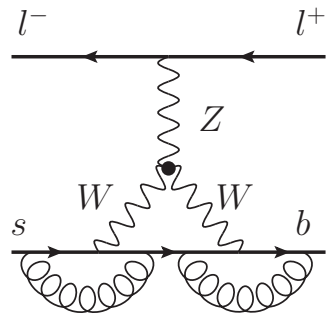

Figure 3: Sample $Z$-boson penguin diagrams contributing to $C_{A}$.

In Fig. 2, the results for the three-loop corrections to $C_{A}^{W}$ are shown as functions of $y=M_{W} / m_{t}$. The dashed and solid lines correspond to the $y \rightarrow 0$ and $y \rightarrow 1$ expansions, respectively. For the charm quark (left panel of Fig. 2) as well as for the top quark contribution (right panel of Fig. 2), the two different expansions show a nice overlap. Considering the thin lines in Fig. 2, which represent lower terms in the expansions, they indicate a good convergence for both expansions. In the physical region of $y$ (yellow band), the expansion arround $m_{t}=M_{W}$ is sufficient.

The second type of diagrams contributing to $C_{A}$ are the Z-boson penguins, sample diagrams are shown in Fig. 3. For those contributions it is necessary to introduce an electroweak counterterm. In addition, at the three-loop level one encounters diagrams with triangle quark loops that involve axial current couplings to the Z-boson. In these cases, one needs to be careful about the treatment of $\gamma_{5}$. In this work we follow the strategy of trace evanescent operators [34] and cross-checked it against Larin's method [35]. In the left panel of Fig. 4 the sum of the three-loop contributions to $C_{A}^{Z}$ are shown. The two expansions for $y \rightarrow 0$ (dashed line) and $y \rightarrow 1$ (solid line) again overlap. In the physical region (yellow band), the expansion $y \rightarrow 1$ is again sufficient.

In the right panel of Fig. 4 the matching scale dependence of $\left|C_{A}\right|^{2}$ is shown. In the SM the branching ratio is proportional to $\left|C_{A}\right|^{2}$ (cf. Eq. (2.3)). The dotted, dashed and solid curves in the right panel of Fig. 4 show the leading order (LO), next-to-leading-order (NLO) and the new next- 

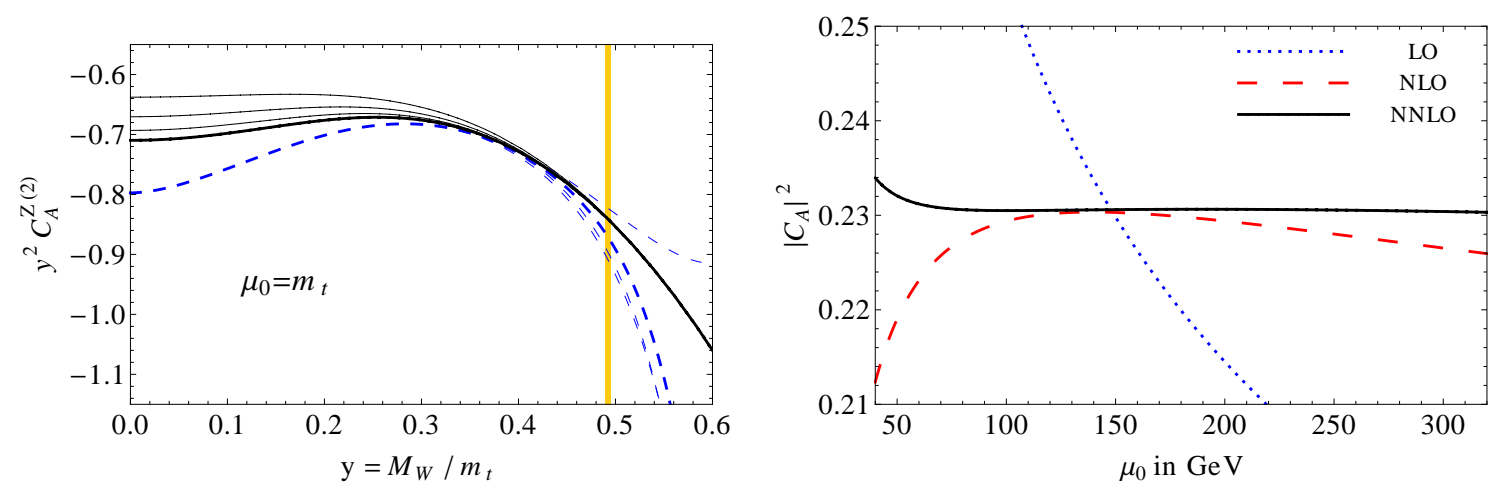

Figure 4: Left: $y^{2} C_{A}^{Z,(2)}$ as a function of $y=M_{W} / m_{t}$. Right: Matching scale dependence of $\left|C_{A}\right|^{2}$ at LO, NLO and NNLO in QCD without higher order corrections in EW interactions.

to-next-to-leading order (NNLO) results. The variation of the matching scale for $\frac{1}{2} m_{t}<\mu_{0}<2 m_{t}$ amounts to around $1.8 \%$ at the NLO. With the new three-loop QCD corrections the uncertainty gets reduced to less than $0.2 \%$.

The results shown so far are at LO in EW interactions. The combination with the recently calculated NLO EW corrections [16] and the RGE running from Refs. [36, 37] leads to the branching ratio $\overline{\mathscr{B}}\left(B_{s} \rightarrow \mu^{+} \mu^{-}\right)=(3.65 \pm 0.23) \times 10^{-9}$. Details on the numerical analysis can be found in Ref. [38].

\section{3. $\bar{B} \rightarrow X_{s} \gamma$ in the $2 \mathrm{HDM}$ at NNLO in QCD}

For $\mathscr{B}\left(\bar{B} \rightarrow X_{s} \gamma\right)$ in the 2HDM type-II we calculate the three-loop QCD corrections to the Wilson coefficients of the operators

$$
\begin{aligned}
& Q_{7}=\frac{e}{16 \pi^{2}} m_{b}\left(\bar{s}_{L} \sigma^{\mu v} b_{R}\right) F_{\mu v}, \\
& Q_{8}=\frac{g}{16 \pi^{2}} m_{b}\left(\bar{s}_{L} \sigma^{\mu v} T^{a} b_{R}\right) G_{\mu v}^{a} .
\end{aligned}
$$

The three-loop matching for $C_{7}$ and $C_{8}$ in 2HDMs works similar to the SM matching [28], details of the calucaltion can be found in Ref. [18].

The interaction Lagrangian for the charged Higgs boson with quarks reads

$$
\mathscr{L}_{H^{+}}=\left(2 \sqrt{2} G_{F}\right)^{1 / 2} \sum_{i, j=1}^{3} \bar{u}_{i}\left(A_{u} m_{u_{i}} V_{i j} P_{L}-A_{d} m_{d_{j}} V_{i j} P_{R}\right) d_{j} H^{+}+\text {h.c. } .
$$

In the type-II model the coefficients $A_{d}$ and $A_{u}$ are given by

$$
A_{u}=-\frac{1}{A_{d}}=\frac{1}{\tan \beta},
$$

where $\tan \beta$ is the ratio of the vacuum expectation values of the two Higgs doublets. In analogy to the calculation of $C_{A}$, we have to consider vacuum integrals with two mass scales $\left(M_{H^{+}}\right.$and $\left.m_{t}\right)$. Sample diagrams for $C_{7}$ up to three-loops are shown in Fig. 5. At the one- and two-loop level, 
(a)

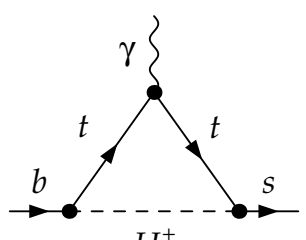

$H^{ \pm}$ (b)

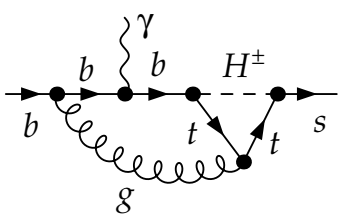

(c)

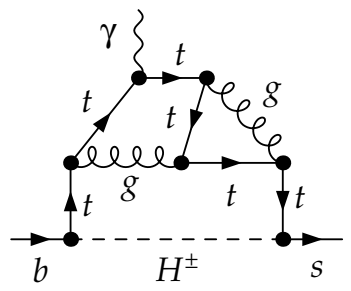

Figure 5: Sample Feynman diagrams contributing to $C_{7}$ at one-, two- and three-loop order.

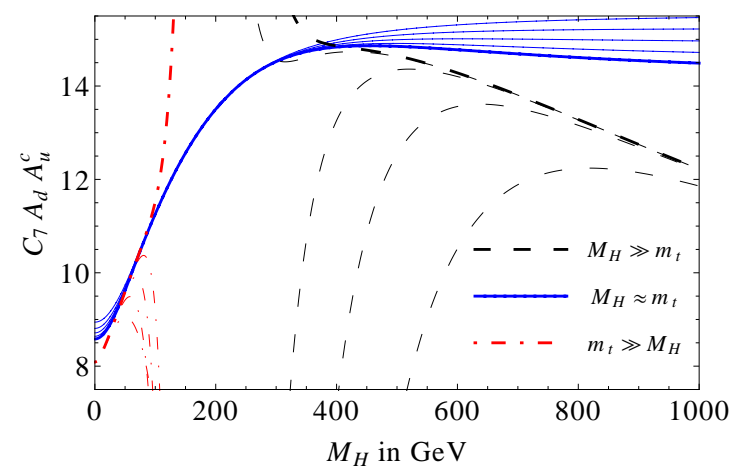

(a)

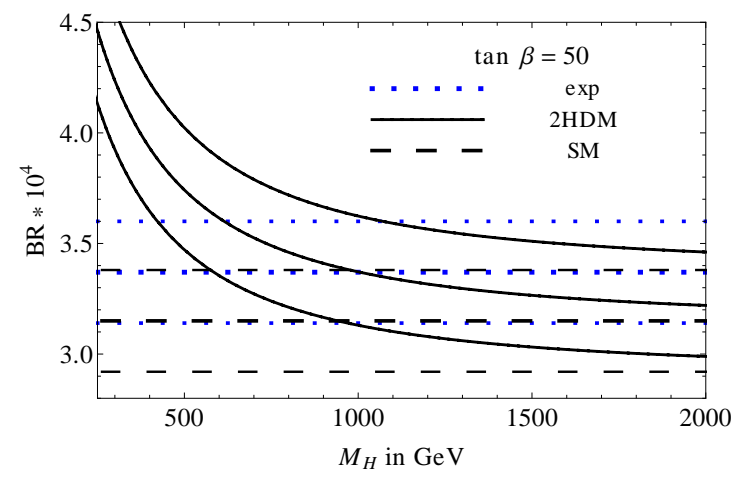

(b)

Figure 6: Left: Three-loop coefficient $C_{7, A_{d} A_{u}^{*}}$ as a function of $M_{H^{+}}$for the three different expansions. Right: $\mathscr{B}\left(\bar{B} \rightarrow X_{s} \gamma\right)$ in dependence of $M_{H^{+}}$. Solid and dashed lines correspond to the NNLO 2HDM and SM predictions, the dotted curves represent the experimental average, all with their respective $1 \sigma$ uncertainty band.

the calculation can be performed exactly, and one obtains $C_{7}$ as a function of $m_{t} / M_{H^{+}}$[39-42]. At the three-loop level, we proceed as in Section 2 and consider expansions around $m_{t} \approx M_{H^{+}}$, for $m_{t} \ll M_{H^{+}}$and for $m_{t} \gg M_{H^{+}}$. In the left panel of Fig. 6 the part of the three-loop correction to $C_{7}$ proportional to $A_{d} A_{u}^{*}$ is shown in dependence of the charged Higgs boson mass. The thickdashed, solid and dash-dotted lines show the results for $M_{H^{+}} \rightarrow \infty, M_{H^{+}} \approx m_{t}$ and $M_{H^{+}} \rightarrow 0$, respectively. There is an overlap between the expansions for $M_{H^{+}} \rightarrow 0$ and $M_{H^{+}} \approx m_{t}$ as well as for the expansions $M_{H^{+}} \approx m_{t}$ and $M_{H^{+}} \rightarrow \infty$, which implies that the expansions are sufficient to obtain $C_{7}$ for any value $M_{H^{+}}$. For the second part of $C_{7}$ (proportional to $A_{u} A_{u}^{*}$ ) and $C_{8}$ the results look very similar.

For the calculation of the branching ratio we use all known NNLO QCD ingredients from Ref. $[19,20]$. In the $2 \mathrm{HDM}$ the branching ratio depends on $\tan \beta$ and $M_{H^{+}}$. For the type-II model the branching ratio is almost independent of $\tan \beta$, for $\tan \beta \gtrsim 2$. For $\tan \beta \lesssim 2$ the branching ratio is strongly enhanced and much higher than the experimental results. In the right panel of Fig. 6 the branching ratio is shown as a function of the charged Higgs mass for $\tan \beta=50$ (solid lines). In 
addition, the SM prediction (dashed lines) and the experimental average (dotted lines) are shown. The middle lines represent the central values, while the upper and lower ones are shifted by $\pm 1 \sigma$. From Fig. 6 one can extract the following limit for $M_{H^{+}}$[18],

$$
M_{H^{+}} \geq 360 \mathrm{GeV} \text { at } 95 \% \text { C.L., }
$$

where the experimental average $\left.\mathscr{B}\left(\bar{B} \rightarrow X_{s} \gamma\right)\right|_{E_{\gamma}>1.6 \mathrm{Gev}}=(3.43 \pm 0.22) \times 10^{-4}$ from the HFAG web page [43] has been used.

\section{Conclusion}

In this contribution the calculation of the Wilson coefficients $C_{A}$ in the $\mathrm{SM}$ and $C_{7}$ in $2 \mathrm{HDM}$ to NNLO in QCD were discussed. Inclusion of the three-loop corrections leads in both cases to a significant reduction of the matching scale dependence. Together with the NLO EW corrections [16] for $C_{A}$, the $\mathrm{SM}$ prediction is given by $\overline{\mathscr{B}}\left(B_{s} \rightarrow \mu^{+} \mu^{-}\right)=(3.65 \pm 0.23) \times 10^{-9}$ [38]. In the $2 \mathrm{HDM}$ type-II a lower limit of $M_{H^{+}} \geq 360 \mathrm{GeV}$ at $95 \%$ C.L. [18] for the charged Higgs boson mass has been obtained.

\section{Acknowledgements}

I thank Mikołaj Misiak and Matthias Steinhauser for the fruitful collaboration and carefully reading this manuscript. I also thank Franziska Schissler for helpful comments. This work was supported by the DFG through the SFB/TR 9 "Computational Particle Physics" and the Graduiertenkolleg "Elementarteilchenphysik bei höchster Energie und höchster Präzision".

\section{References}

[1] R. Aaij et al. (LHCb Collaboration), Phys. Rev. Lett. 110 (2013) 021801 [arXiv:1211.2674].

[2] R. Aaij et al. (LHCb Collaboration), Phys. Rev. Lett. 111, 101805 (2013) [arXiv:1307.5024].

[3] S. Chatrchyan et al. (CMS Collaboration), Phys. Rev. Lett. 111, 101804 (2013) [arXiv:1307.5025].

[4] CMS and LHCb Collaborations, conference report CMS-PAS-BPH-13-007, LHCb-CONF-2013-012, http://cds.cern.ch/record/1564324.

[5] V. M. Abazov et al. (D0 Collaboration), Phys. Lett. B 693 (2010) 539 [arXiv:1006.3469].

[6] T. Aaltonen et al. (CDF Collaboration), Phys. Rev. Lett. 107 (2011) 191801 [arXiv:1107.2304].

[7] S. Chatrchyan et al. (CMS Collaboration), JHEP 1204 (2012) 033 [arXiv:1203.3976].

[8] R. Aaij et al. (LHCb Collaboration), Phys. Rev. Lett. 108 (2012) 231801 [arXiv:1203.4493].

[9] G. Aad et al. (ATLAS Collaboration), Phys. Lett. B 713 (2012) 387 [arXiv:1204.0735].

[10] P. Dimopoulos et al. (ETM Collaboration), JHEP 1201 (2012) 046 [arXiv:1107.1441].

[11] C. McNeile, C. T. H. Davies, E. Follana, K. Hornbostel and G. P. Lepage (HPQCD Collaboration), Phys. Rev. D 85 (2012) 031503 [arXiv:1110.4510].

[12] A. Bazavov et al. (Fermilab Lattice and MILC Collaborations), Phys. Rev. D 85 (2012) 114506 [arXiv:1112.3051]. 
[13] F. Bernardoni et al., (ALPHA Collaboration), Nucl. Phys. Proc. Suppl. 234 (2013) 181 [arXiv:1210.6524].

[14] N. Carrasco et al. (ETMC Collaboration), PoS ICHEP 2012 (2012) 428 [arXiv:1212.0301].

[15] R. J. Dowdall et al. (HPQCD Collaboration), Phys. Rev. Lett. 110 (2013) 222003 [arXiv:1302.2644].

[16] C. Bobeth, M. Gorbahn and E. Stamou, arXiv:1311.1348 [hep-ph].

[17] T. Hermann, M. Misiak and M. Steinhauser, arXiv:1311.1347 [hep-ph].

[18] T. Hermann, M. Misiak and M. Steinhauser, JHEP 1211 (2012) 036 [arXiv:1208.2788].

[19] M. Misiak and M. Steinhauser, Nucl. Phys. B 764 (2007) 62 [hep-ph/0609241].

[20] M. Misiak, et al., Phys. Rev. Lett. 98 (2007) 022002 [hep-ph/0609232].

[21] K. De Bruyn, R. Fleischer, R. Knegjens, P. Koppenburg, M. Merk, A. Pellegrino and N. Tuning, Phys. Rev. Lett. 109 (2012) 041801 [arXiv:1204.1737].

[22] T. Inami and C. S. Lim, Prog. Theor. Phys. 65 (1981) 297 [Erratum-ibid. 65 (1981) 1772].

[23] G. Buchalla and A. J. Buras, Nucl. Phys. B 398 (1993) 285.

[24] G. Buchalla and A. J. Buras, Nucl. Phys. B 400 (1993) 225.

[25] M. Misiak and J. Urban, Phys. Lett. B 451 (1999) 161 [hep-ph/9901278].

[26] G. Buchalla and A. J. Buras, Nucl. Phys. B 548 (1999) 309 [hep-ph/9901288].

[27] J. Grigo, J. Hoff, P. Marquard and M. Steinhauser, Nucl. Phys. B 864 (2012) 580 [arXiv:1206.3418].

[28] M. Misiak and M. Steinhauser, Nucl. Phys. B 683 (2004) 277 [hep-ph/0401041].

[29] P. Nogueira, J. Comp. Phys. 105 (1993) 279.

[30] T. Seidensticker, hep-ph/9905298;

R. Harlander, T. Seidensticker and M. Steinhauser, Phys. Lett. B 426 (1998) 125 [hep-ph/9712228].

[31] V. A. Smirnov, “Analytic tools for Feynman integrals," Springer Tracts Mod. Phys. 250, 1 (2012).

[32] M. Steinhauser, Comput. Phys. Commun. 134 (2001) 335 [hep-ph/0009029].

[33] J.A.M. Vermaseren, Symbolic Manipulation with FORM, Computer Algebra Netherlands, Amsterdam, 1991.

[34] M. Gorbahn and U. Haisch, Nucl. Phys. B 713 (2005) 291 [hep-ph/0411071].

[35] S. A. Larin, Phys. Lett. B 303 (1993) 113 [hep-ph/9302240].

[36] C. Bobeth, P. Gambino, M. Gorbahn and U. Haisch, JHEP 0404 (2004) 071 [hep-ph/0312090].

[37] T. Huber, E. Lunghi, M. Misiak and D. Wyler, Nucl. Phys. B 740 (2006) 105 [hep-ph/0512066].

[38] C. Bobeth, M. Gorbahn, T. Hermann, M. Misiak, E. Stamou and M. Steinhauser, arXiv:1311.0903 [hep-ph].

[39] M. Ciuchini, G. Degrassi, P. Gambino and G. F. Giudice, Nucl. Phys. B 527 (1998) 21 [hep-ph/9710335].

[40] F. Borzumati and C. Greub, Phys. Rev. D 58 (1998) 074004 [hep-ph/9802391], Phys. Rev. D 59 (1999) 057501 [hep-ph/9809438].

[41] P. Ciafaloni, A. Romanino and A. Strumia, Nucl. Phys. B 524 (1998) 361 [hep-ph/9710312].

[42] C. Bobeth, M. Misiak and J. Urban, Nucl. Phys. B 567 (2000) 153 [hep-ph/9904413].

[43] http://www.slac.stanford.edu/xorg/hfag/ 